\title{
Time-resolved granular dynamics of a rotating drum in a slumping regime as revealed by speckle visibility spectroscopy
}

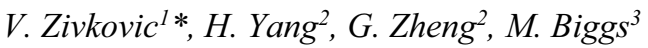 \\ ${ }^{1}$ School of Chemical Engineering and Advanced Materials, Newcastle University, NE1 7RU, United Kingdom \\ ${ }^{2}$ a. School of Optical-Electrical and Computer Engineering, b. school of medical instrument and food engineering, c. Shanghai Key Lab \\ of Modern Optical System, d. Engineering Research Center of Optical Instrument and System, Ministry of Education, University of \\ Shanghai for Science and Technology, Shanghai, 200093, China \\ ${ }^{3}$ School of Science, Loughborough University, LE11 3TU, United Kingdom
}

\begin{abstract}
Granular materials in rotating drums are of wide interest not only because of their extensive use in the industrial contexts, but also as model systems in the study of natural disasters, such as avalanches or landslides. Most of available experimental methods are restricted to surface layer flows and dilute systems whilst the remainder can only resolve the granular dynamics to a fine scale with relatively poor temporal resolution or vice versa. In contrast, speckle visibility spectroscopy (SVS) is able to resolve the average of the three components of motion of grains in dense systems in small volume of granular media several layer deep with spatio-temporal resolutions that allow the probing of the granular micro-dynamics. We have used this technique to study granular dynamics of surface avalanche flow in the slumping regime using both spherical glass and irregular sand particles. Although results are very similar, we determined that visually observed compaction at the beginning of avalanche process for irregular sand particles influence time evolution of the particle fluctuation velocity during avalanches.
\end{abstract}

\section{Introduction}

Granular flows in rotating drums are of wide interest not only as model systems in the study of the physics of granular media, but also because of their extensive use in the chemical, minerals, pharmaceutical and food industry where they are employed to effect process as diverse as mixing and granulation. It is well known that as the drum rotational speed increases, the granular flow takes on one of a number of regimes [1], including slumping, which occurs at lower rotational speeds with discrete avalanches and is of wide relevance to industrial processes as well as environmental problems such as dune migration, sediment transport, landslides and natural avalanches in general [2,3]. However, the overwhelming majority of the studies of rotating drum systems have focussed on special case of steady continuous surface flow at constant dynamic angle (rolling and cascading regime) mostly due to their industrial importance. Although non-spherical particles are rather common both in industrial and environmental context, experimental investigations of non-spherical and irregular particles behaviour in rotating drums are scarce and rather limited in scope. For example, there is no experimental measurement of time resolved particle dynamics of transient flowing layer during discrete avalanches of slumping regime for non-spherical particles and even only few studies for spherical $[4,5]$.

There are a range of experimental methods available for the study of the granular dynamics in rotating drums, including particle image velocimetry (PIV) [4,6-8], particle tracking velocimetry (PTV) [9,10], PEPT $[11,12]$, magnetic resonance imaging $[13,14]$, and x-ray microtomography [15]. Some of these are restricted to two components of motion (PIV and PTV) whilst the remainder can only resolve the granular dynamics to a fine scale with relatively poor temporal resolution or vice versa. In contrast, diffusing-wave spectroscopy [16], a dynamic light scattering technique, is able to resolve the average of the three components of motion of grains in dense systems with very fine spatiotemporal resolutions $[17,18]$ that allow the probing of the dynamics of avalanches. However, it is based on temporal correlation functions calculated as a time average, so it is not appropriate for systems with transient dynamics. In such cases, the related method of speckle visibility spectroscopy (SVS) [5,19-21] can be used.

In this paper, we report the SVS-based study of the avalanching of particles in a rotating drum under the slumping regime. After introducing the SVS method and the rotating granular drum flow system to which it has been applied here, we report time resolved avalanche dynamics results for spherical glass and non-spherical sand particles followed by their qualitative comparison.

\section{Experimental details}

\subsection{Experimental setup}

The results reported here were obtained in a cylindrical drum, Fig. 1, whose inner diameter, D, and length, $\mathrm{L}$, are

\footnotetext{
* Corresponding author: vladimir.zivkovic@ncl.ac.uk
} 
$142 \mathrm{~mm}$ and $200 \mathrm{~mm}$ respectively. The rotating drum, which is made of clear Plexiglas to permit optical access, was filled with granular material. The drum was placed on a pair of rollers turned by a DC motor in 0.1-2.3 revolutions per minute (RPM). Points on the free surface of the granular material were studied here by laser illumination as shown in Fig. 1, some at the top of the inclined free surface, whilst others were located at the bottom of this surface. Two granular material were considered: spherical glass particles $(500-600 \mu \mathrm{m}$ diameter range) and glass sand grains $(1.0-1.5 \mathrm{~mm})$. Based on shape descriptors obtained through image analysis, the sand grains would be categorised as subangular particles in the qualitative shape measure classification [22].

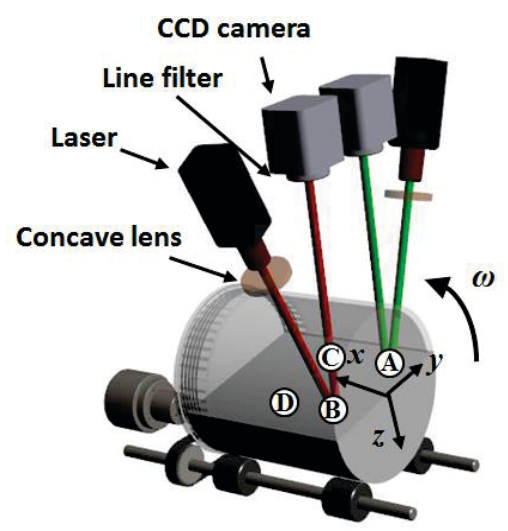

Fig. 1. Schematic of the experimental setup.

\subsection{SVS methodology}

The speckle visibility spectroscopy involves illuminating the avalanching free surface spots of the granular materials with lasers. In case of sand particles we have used synchronized double speckle visibility spectroscopy [23] using two monochromatic lasers of wavelengths $671 \mathrm{~nm}$ and $532 \mathrm{~nm}$ while only $532 \mathrm{~nm}$ laser was used for glass particles study. The light beams were passed through a concave lens before normally illuminating a spots of around $10 \mathrm{~mm}$ diameter on the inclined free surface of the granular material. The photons that emerged from the granular material after diffusing within it form a speckle pattern that was detected using high speed line scan CCD cameras (up to $50 \mathrm{kHz}$ ). The cameras were placed with its optical axis normal to the drum and some 350 to $400 \mathrm{~mm}$ from it, such that the ratio of pixel to speckle size was about 0.5 . In the absence of any motion of the particles, the scattered light is characterised by a constant intensity as illustrated by the example in Fig.2(a). Collective motion of particles as a rigid body leads to the spatial and temporal fluctuations of the scattered light resulting in random intensity of lights across the pixels, as illustrated by the example in Fig. 2(b). However, only the relative motion of particles between each other's results in blurring of captured images and for a given exposure time, the faster the dynamics of the grains, the more the speckle image is blurred and the lower the contrast - this enables the capture of rapid changes in the granular material with time such as that which occurs in an avalanche as illustrated in the example of Fig. 2(c). This variation in intensity can be quantified by the variance of the intensity [19]

$$
V_{2}(T) \propto\left\langle I^{2}\right\rangle_{T}-\langle I\rangle^{2}
$$

where $\langle\cdots\rangle_{\mathrm{T}}$ denotes the average over pixels exposed for a duration $T$. The proportionality constant of $V_{2}(T)$ is set by the laser intensity and the ratio of speckle to pixel size (i.e. it is set-up dependent). It can, however, be eliminated by considering the variance ratio $V_{2}(2 T) / V_{2}(T)$ [19]. For diffusely backscattered light from particles moving with a random ballistic motion, where the power spectrum is Lorentzian [24], the theory of SVS [19] gives the variance ratio as

$$
\frac{V_{2}(2 T)}{V_{2}(T)}=\frac{e^{-4 \Gamma T}-1+4 \Gamma T}{4\left(e^{-2 \Gamma T}-1+2 \Gamma T\right)}
$$

where $\Gamma=4 \pi \delta v / \lambda$. The mean fluctuation in the speed of the particles, $\left(\delta v^{2}\right)$, which is equal to the collision velocity and directly related to the so-called granular temperature [36], can be obtained by inverting Eq. (2), as given in Fig. 2(d) which was obtained from the raw data shown in Fig 2(c). Comparison of these two subfigures clearly shows that only the relative motion of particles during avalanching is detected while the rigid body motion before and after avalanching event is shown with the near zero collisional velocity.

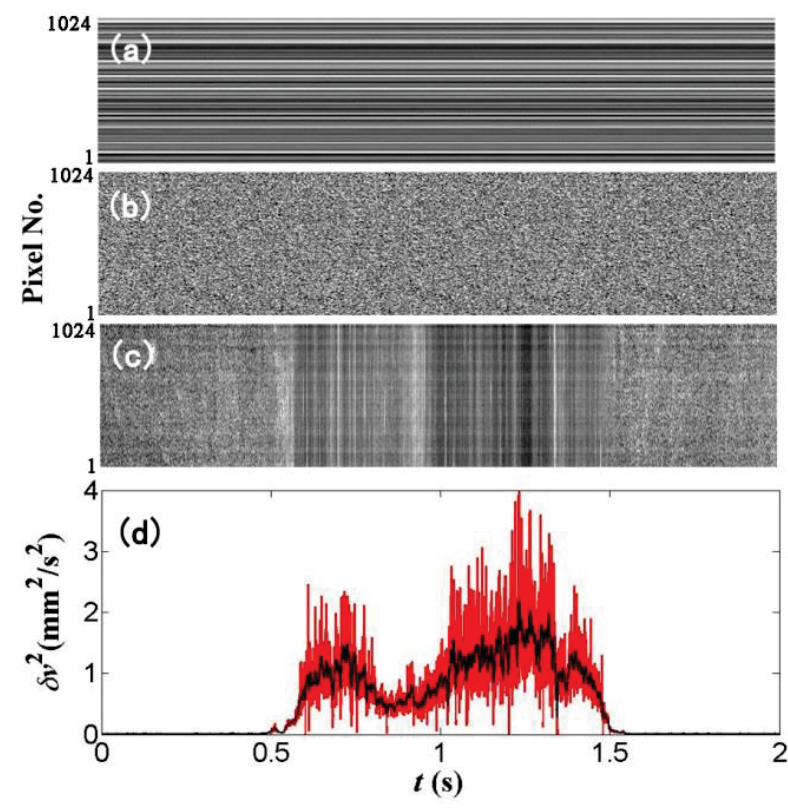

Fig. 2. Example of SVS results and analysis for sand particles in the drum: (a) intensity across the CCD pixels vs. time when the drum is stationary; (b) intensity across the CCD pixels vs. time when the drum is rotating at a speed of 1.53 RPM and no avalanche event occurs; (c) the same as (b) except an avalanche event has occurred in the period of around 0.5-1.5 s; (d) The grey (red online) line represents the raw particle fluctuation velocity $\left(\delta v^{2}\right)$ obtained from the data in (c), whilst the black line represents the data after being subject to a Butterworth low pass filter to reduce the noise.

\section{Results and discussion}

\subsection{Avalanche statistics}

Fig. 3 shows the typical SVS-derived time trace of the granular temperature, $\left(\delta v^{2}\right)$, over several avalanche events for both spherical glass and irregular sand particles. Results for the glass particles, Fig. 3(a), are from the top point on the inclined surface at a drum at 
rotational speed of 0.15 RPM along with a filtered version thereof, which removes CCD camera related noise. Looking at a single avalanche event shows that the particle velocity fluctuation accelerates progressively to a maximum before similarly decelerating. The avalanche duration, $t_{d}$, rest time, $t_{r}$, and the peak of the particle velocity fluctuation, $\left(\delta v_{p}^{2}\right)$, can be straightforwardly measured from the filtered trace for many slumping cycles to enable good statistics (at least 100 avalanches analyzed).
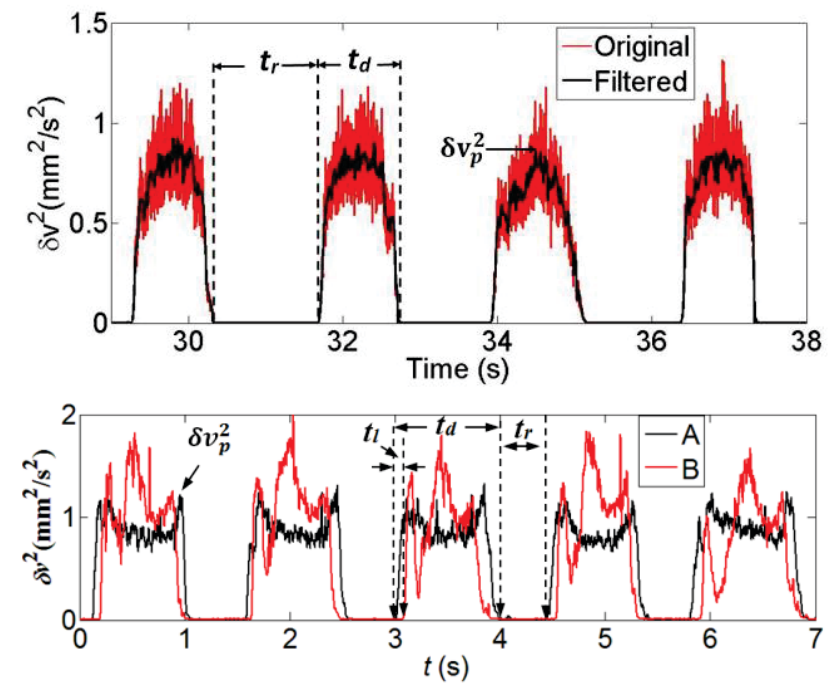

Fig. 3. Time trace of granular temperature, $\left(\delta v^{2}\right)$ over several avalanches for (a) glass particles in a drum rotating at a speed of 0.15 RPM and (b) sand grains in drum rotating at 1.53 RPM.

The synchronized granular temperature time trace of sand grains over five slumping cycles at a drum speed of 1.53 RPM from the top, point A, and bottom, point B, of the inclined surface is given in Fig. 3(b). Looking at a single avalanche event for the point A shows that the particle fluctuation velocity accelerated quickly then reaching plateau for some time before decelerating quickly again in contrast to spherical glass particle experiments where this is more progressively transition. This is probably related to observed compaction of the drum at the beginning of avalanching process followed by expansion afterwards as indicated in plot of relative area of the drum during typical avalanche, Fig. 4. The compaction was not detected in case of spherical glass beads confirming that the particle shape is important for granular compaction [25].

Another difference are two peaks of the SVS signal at the beginning and the end of the avalanching period for point B albeit sometimes this is not very clear. Again visual observation (not shown here) give possible explanation as a small mound forms nearly at the centre of the avalanching surface approximately halfway through the avalanche event. Therefore, the bottom point (B) granular temperature shows some peaks and drops midway through the avalanche period due to the passing of this mound, while the top point A is not as influenced and has more stable signal.

Avalanching statistics in the middle of drum (not shown here) is also more stable and the granular temperatures are lower in comparison with near wall measurements shown in Fig. 3(b). This is in line with the previous reports that the flow velocity of the grains is lower in the middle than that near the front wall of the drum for the wall effect $[26,27]$. The stability of the signals would indicate that the visually observed mound is appearing only near the end walls and does not influence the avalanche dynamics in the middle of the drum.

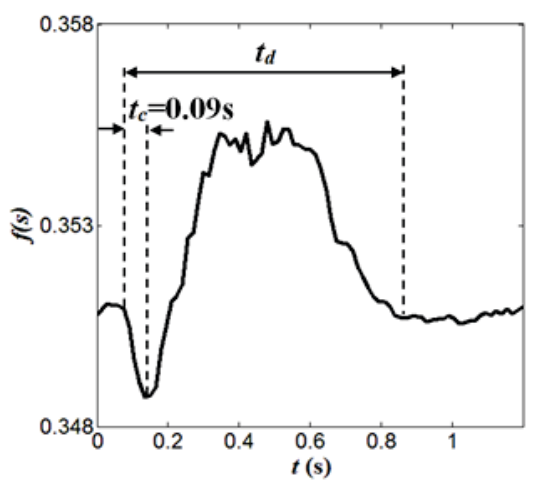

Fig. 4. The evolution of relative area, $f(s)$, during avalanching from the movies of the front face at a drum speed of 1.53 RPM.
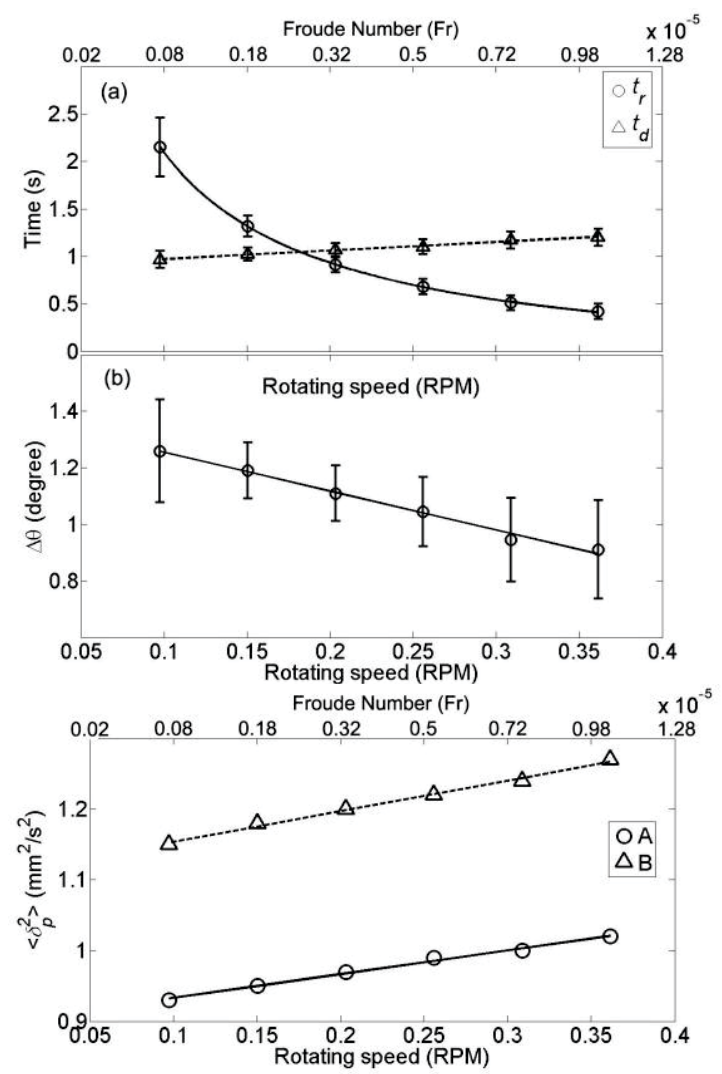

Fig. 5. (a) The average rest time, $t_{r}$, and the average avalanche duration time, $t_{d}$; (b) $\Delta \theta$ by obtained by SVS analysis and (c) the average of the peak of particle fluctuation speed, $\left(\delta v_{p}{ }^{2}\right)$, of the avalanches for the point A (top) and B (bottom) as a function of the rotating speeds and Froude number for spherical glass particles.

\subsection{Variation of slumping features with drum speed}

Figures 5 and 6 shows variation of various avalanche statistics with drum speed and corresponding Froude number for glass and sand particles. The trends are quite similar but with some differences in the values. Obvious one is that drum with sand particles is operating at higher drum speed and consequently Froude number which in 
in line with previous studies that transition to rolling regime is highly influence by particle shape [3]. Another is that the difference between upper and lower angle of repose is almost 5 times bigger for sand particles and consequently the rest time between avalanches is also approximately 5 times bigger. However, the trends of various avalanche characteristics with the drum speed are very similar for both particles such as constant avalanche duration, decrease of the difference between upper and lower angle of repose and linear increase of granular temperature with the drum speed.
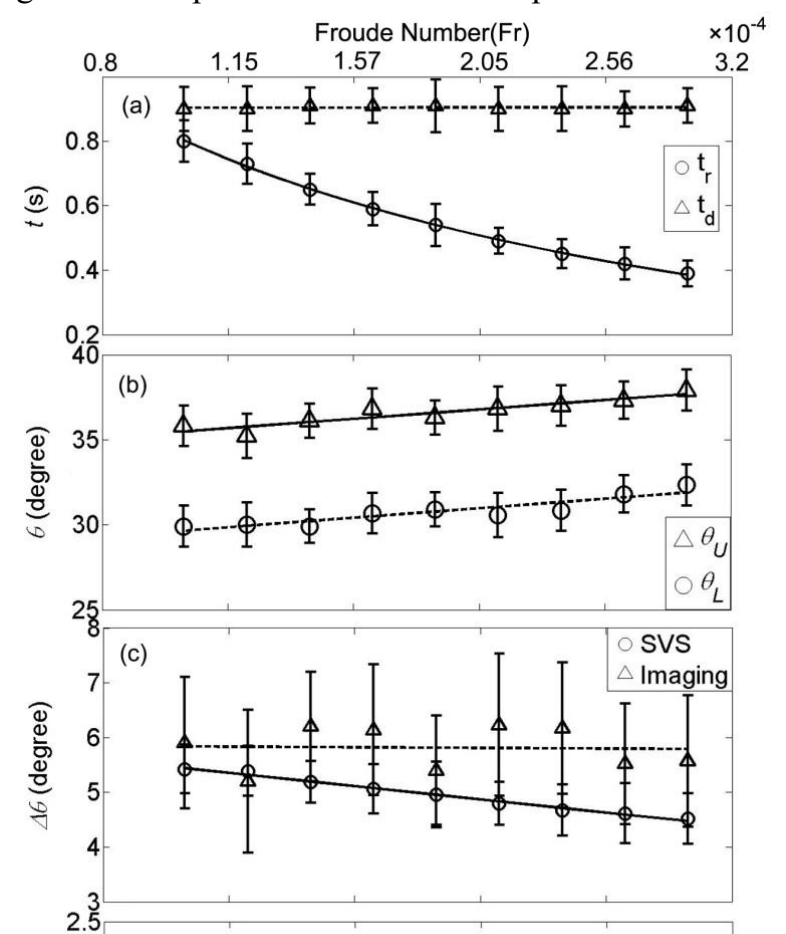

(d)

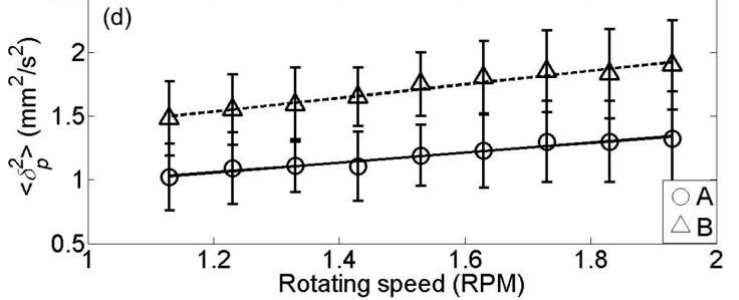

Fig. 6 (a) The average rest time, $t_{r}$, and the average avalanche duration time, $t_{d}$; (b) upper and lower angle of repose from image analysis; (c) $\Delta \theta$ by SVS (open circle) and imaging technique (triangle) and (d) the average of the peak of particle fluctuation speed, $\left(\delta v_{p}^{2}\right)$, of the avalanches for the point $\mathrm{A}$ (top) and B (bottom) as a function of the rotating speeds and Froude number for sand grains.

\section{Conclusion}

Our study showed that the speckle visibility spectroscopy is able to measure time resolved granular micro-dynamics of intermittent surface avalanche flow inside rotating drum operating in a slumping regime. There are some differences in time evolution of granular temperature between glass and sand particles probably due to visually observed compaction and consequent formation of the mound as a result of irregular shape of sand grains.

\section{References}

[1] H. Henein, J. K. Brimacombe, and A. P. Watkinson, Metall. Trans. B 14, 191 (1983).

[2] G. Seiden and P. J. Thomas, Rev. Mod. Phys. 83, 1323 (2011).

[3] J. Mellmann, Powder Technol. 118, 251 (2001).

[4] S. C. du Pont, R. Fischer, P. Gondret, B. Perrin, and M. Rabaud, Phys. Rev. Lett. 94, 048003 (2005).

[5] H. Yang, R. Li, P. Kong, Q. C. Sun, M. J. Biggs, and V. Zivkovic, Phys. Rev. E 91, 042206 (2015).

[6] S. H. Chou and S. S. Hsiau, Powder Technol. 226, 99 (2012).

[7] H. Komossa, S. Wirtz, V. Scherer, F. Herz, and E. Specht, Powder Technol. 264, 96 (2014).

[8] H. T. Chou, S. H. Chou, and S. S. Hsiau, Powder Technol. 252, 42 (2014).

[9] R. Mueller and P. Kleinebudde, Powder Technol. 173, 51 (2007).

[10] N. Jain, J. M. Ottino, and R. M. Lueptow, Phys. Fluids 14, 572 (2002).

[11] D. J. Parker, A. E. Dijkstra, T. W. Martin, and J. P. K. Seville, Chem. Eng. Sci. 52, 2011 (1997).

[12] S. Y. Lim, J. F. Davidson, R. N. Forster, D. J. Parker, D. M. Scott, and J. P. K. Seville, Powder Technol. 138, 25 (2003).

[13] K. Yamane, M. Nakagawa, S. A. Altobelli, T. Tanaka, and Y. Tsuji, Phys. Flluids 10, 1419 (1998).

[14] C. R. Müller, D. J. Holland, A. J. Sederman, M. D. Mantle, L. F. Gladden, and J. F. Davidson, Powder Technol. 183, 53 (2008).

[15] G. T. Seidler, G. Martinez, L. H. Seeley, K. H. Kim, E. A. Behne, S. Zaranek, B. D. Chapman, S. M. Heald, and D. L. Brewe, Phys. Rev. E 62, 8175 (2000).

[16] D. J. Pine, D. A. Weitz, P. M. Chaikin, and E. Herbolzheimer, Phys. Rev. Lett. 60, 1134 (1988).

[17] V. Zivkovic, M. J. Biggs, D. H. Glass, and L. Xie, Adv. Powder Technol. 20, 227 (2009).

[18] V. Zivkovic, K. Berry, D. H. Glass, and M. J. Biggs, in POWDERS AND GRAINS 2013: Proceedings of the 7th International Conference on Micromechanics of Granular Media (AIP Publishing, 2013), pp. 1266.

[19] R. Bandyopadhyay, A. S. Gittings, S. S. Suh, P. K. Dixon, and D. J. Durian, Rev. Sci. Instrum. 76, 093110 (2005).

[20] H. Katsuragi, A. R. Abate, and D. J. Durian, Soft Matter 6, 3023 (2010).

[21] H. Yang, G. L. Jiang, H. Y. Saw, C. Davies, M. J. Biggs, and V. Zivkovic, Chem. Eng. Sci. 146, 1 (2016).

[22] F. Altuhafi, C. O'sullivan, and I. Cavarretta, J Geotech. Geoenviron. 139, 1290 (2012).

[23] R. Li, H. Yang, G. Zheng, B. F. Zhang, M. L. Fei, and Q. C. Sun, Powder Technol. 295, 167 (2016).

[24] P. A. Lemieux and D. J. Durian, JOSA A 16, 1651 (1999).

[25] N. Vandewalle, G. Lumay, O. Gerasimov, and F. Ludewig, Eur. Phys. J. E 22, 241 (2007).

[26] N. A. Pohlman, J. M. Ottino, and R. M. Lueptow, Phys. Rev. E 74, 031305 (2006).

[27] M. Arntz, W. K. Den Otter, H. H. Beeftink, R. M. Boom, and W. J. Briels, Granular matter 15, 25 (2013). 